\title{
Experience with Temporary Centrifugal Pump Bi-ventricular Assist Device for Pediatric Acute Heart Failure: Comparison with ECMO
}

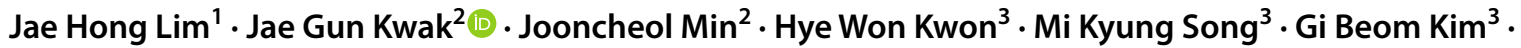 \\ Eun Jung Bae ${ }^{3}$. Woong-Han Kim² ${ }^{2}$ Jeong Ryul Lee ${ }^{2}$
}

Received: 2 December 2019 / Accepted: 8 July 2020 / Published online: 27 August 2020

(c) Springer Science+Business Media, LLC, part of Springer Nature 2020

\begin{abstract}
Though ventricular assist devices (VADs) are an important treatment option for acute heart failure, an extracorporeal membrane oxygenator (ECMO) is usually used in pediatric patients for several reasons. However, a temporary centrifugal pump-based Bi-VAD might have clinical advantages versus ECMO or implantable VADs. From January 2000 to July 2018, we retrospectively reviewed 36 pediatric patients who required mechanical circulatory support (MCS) for acute heart failure. Cases with postoperative MCS were excluded. Since 2016, we have tried to immediately add a right VAD rather than ECMO, when the patients begin to present features of right heart failure after left VAD support started in cases that the patients' respiratory function did not require an oxygenator. Original diagnoses included dilated cardiomyopathy $(n=18)$, myocarditis $(n=11)$, and others $(n=7)$. Eleven patients were supported by Bi-VAD, and 25 patients were supported by ECMO; of these. Four patients were successfully weaned from VAD, and 10 patients were weaned from ECMO. Eleven patients underwent heart transplantation. Overall, we have $15(41.7 \%)$ early mortalities. There were no significant differences in early mortality, morbidity, and weaning rate between the Bi-VAD group and the ECMO group. During the support, patients with Bi-VADs significantly required fewer platelets and showed less hemolysis than ECMO patients. Patients with myocarditis were successfully weaned from Bi-VAD support and bridged to transplantation thereafter. A temporary centrifugal pump-based Bi-VAD was clinically comparable to ECMO for pediatric patients with acceptable pulmonary function.
\end{abstract}

Keywords Pediatric $\cdot$ Heart failure $\cdot$ Ventricular assist device

This topic was presented in poster session in 55th "The Society of Thoracic Surgeons" annual meeting in San Diego, CA, January 27, 2019-January 29, 2019.

Jae Gun Kwak

switch.surgeon@gmail.com

1 Department of Thoracic and Cardiovascular Surgery, Sejong General Hospital, Bucheon, Gyeong-Gi, Republic of Korea

2 Department of Thoracic and Cardiovascular Surgery, Seoul National University Children's Hospital, College of Medicine, Seoul National University, Seoul, Republic of Korea

3 Department of Pediatrics, Seoul National University Children's Hospital, College of Medicine, Seoul National University, Seoul, Republic of Korea

\section{Introduction}

Mechanical circulatory support (MCS) has become an important treatment option for patients with acute decompensated heart failure that is refractory to other medical therapies [1]. Many studies reported various results of MCS including extracorporeal membrane oxygenation (ECMO) and ventricular assist device (VAD) [1-5]. Some authors have reported that VAD confers a statistically significant increase in survival after heart transplantation over ECMO for children with end-stage heart failure [5-12]. However, most of these studies have focused on implantable VADs.

There are limitations to the use of VADs in pediatric patients due to their diminutive sizes, especially in cases requiring bi-ventricular support. Therefore, historically ECMO has been the traditional course of action for pediatric patients regardless of their respiratory function. A variety of implantable VADs are available worldwide, however, the temporary centrifugal pump VAD system is still used in 
selected cases or specific situations, for examples, in a case that patient's heart failure is anticipated to be recovered fast with just a short-term mechanical support, or in a situation that a sufficient financial support for durable VAD by private of national insurance system is not available. In Korea, implantable VADs were not available before 2017. However, even though "Korean Ministry of Food and Drug Safety" approved to use implantable VADs, we still have many restrictions to apply an implantable VAD because of highly appropriated medical expenses to use with limited indications for being supported by a national insurance system.

The use of biventricular assist devices (Bi-VADs) has been associated with decreased survival rates in many studies $[7,11,13,14]$. In these studies, the addition of right VAD (RVAD) support in patients already supported by left VAD (LVAD) was in response to disease progression, i.e., right ventricular (RV) failure and, as such, was linked to poor patient outcome. However, given this selective application, it remains unclear whether Bi-VAD themselves are a risk factor. In fact, the implantation of a RVAD prior to $\mathrm{RV}$ failure could contribute to better patient outcomes for those with belated RVAD deployment or receiving ECMO support, even in small pediatric patients. Therefore, the goal of this study was to examine the clinical outcomes after BiVAD and ECMO-based interventions for medically intractable acute decompensated heart failure in a young patient population.

\section{Methods}

We enrolled 36 patients from January 2000 to July 2018 who were assisted by MCS for medically intractable acute decompensated heart failure. Baseline and follow-up data were collected from the patients' medical chart. This study was approved by the Seoul National University Hospital Institutional Review Board (IRB) with waived patient consent (IRB number: C-1809-116-974). All procedures were performed in accordance with the principles outlines in the Declaration of Helsinki. We excluded postoperative patients with MCS. All of the patients received temporary centrifugal pump-based Bi-VADs assembled with Bio Pump (Medtronic, Inc., Minneapolis, MN, USA) and RotaFlow (Maquet, Jostra Medizintechnik AG, Hirrlingen, Germany). All of the patients in the Bi-VAD group were centrally cannulated under median sternotomy. Drainage and perfusion catheters were usually cannulated at the left atrium through the right upper pulmonary vein and ascending aorta for the LVAD, and the right atrium and main pulmonary artery for the RVAD. ECMO (veno-arterial only) patients were cannulated either centrally or peripherally (neck or groin vessels) depending on the patient's body weight or situations that patients encountered, for example, emergent status or not, the patient's cardiac operation (chest open) history. Peripheral vascular accessibility was determined by ultrasonography prior to the procedure. If the left ventricle (LV) was not decompressed efficiently, then we placed an additional cannula in left atrium and ventricle through right upper pulmonary vein or left atrial appendage.

We divided the patients into two cohorts, the Bi-VAD group and ECMO group, according to which device the patients were supported with initially. Outcome data were compared and analyzed for statistical significance. We also looked at patient outcomes over time (before and after 2010).

\section{Criteria for Bi-VAD Implantation}

Since 2016, 6 of 36 (16.7\%) patients, we have tried to add a RVAD rapidly and immediately for the patients who were already being supported by a LVAD if the patients began to show the features of RV failure. Before 2016, conversion from ECMO to VADs or early RVAD application was not considered or performed as actively as after 2016. We also immediately changed from ECMO to Bi-VAD-based support for patients whose pulmonary function recovered after initial ECMO intervention. Chest X-ray and arterial blood gas analysis (ABGA) were the primary evaluation tools used to assess pulmonary function. If the patient presented a clear lung field on their chest X-ray, a partial pressure of $\mathrm{O}_{2}$ over $90 \mathrm{mmHg}$, and less than $55 \mathrm{mmHg}$ of $\mathrm{CO}_{2}$ on the ABGA with a $60-80 \%$ of fraction of inspired $\mathrm{O}_{2}\left(\mathrm{FiO}_{2}\right)$, we applied RVAD rather than ECMO for RV failure. We opted for a RVAD in patients who had just begun to present clinical symptoms of RV failure (e.g., ascites, pleural effusions, renal or hepatic dysfunction, progression of tricuspid regurgitation, or frequent ventricular arrhythmias) rather than increasing medical treatment efforts (e.g., increasing dosage of milrinone [more than $0.5 \mathrm{mcg} / \mathrm{kg} / \mathrm{min}$ ] or nitric oxide gas [more than $20 \mathrm{mmHg}$ ] or adding additional pharmaceutical agents). For patients already supported by ECMO due to accompanied respiratory problems or easy access through peripheral vessels in an emergency situation, we sought to switch to BiVAD as soon as the patients' pulmonary function improved from its initial deteriorated state or was considered tolerable and no longer required the support of an oxygenator. In the operating room, after LVAD insertion, we monitored RV function using transesophageal echocardiography (change in tricuspid valve regurgitation amount, RV contraction and regional wall motion, and pulmonary hypertension), and central venous pressure. If RV function began to deteriorate, we insufflated nitric oxide gas and infused intravenous milrinone; however, when RV function did not improve despite these efforts, we did not hesitate to apply the RVAD for patients with acceptable lung function. These decisions were reached by a multidisciplinary team of cardiologists, cardiac surgeons and anesthesiologists. 


\section{Anticoagulation Strategy and Other Managements}

After MCS, all of the patients received anticoagulation therapy using intravenous heparin without other oral agents, such as Warfarin or antiplatelet agents. The target for ECMO was 80 to $100 \mathrm{~s}$ for the activated prothrombin time (aPTT) and the activated clotting time (ACT) target was 150 to $180 \mathrm{~s}$. For VAD, the aPTT target is 60 to $80 \mathrm{~s}$ (ACT of 120 to $150 \mathrm{~s}$ ). We checked the aPTT every $4 \mathrm{~h}$ and ACT once daily. Heparin infusion was temporarily suspended in patients with bleeding tendencies until these improved. The circuit of the MCS was checked every $2 \mathrm{~h}$ to detect thrombus formation in the line by nursing staffs, and if we had thrombi in the circuit, we increased the dosage of heparin slowly even though the lab findings were in tolerable range. When the amounts of thrombi increased despite increasing heparin, we considered changing of the circuit.

Recently, we try to keep a patient's consciousness to be awake and to provide an enteral feeding unless a patient complains severe pain. We consult a bed-side physical rehabilitation therapy to the department of rehabilitation to prevent possible musculoskeletal problems caused by a long-term bed-ridden status.

We enlisted 21 patients for transplantation; eight patients in the Bi-VAD group and twelve patients in the ECMO group.

\section{Statistical Analysis}

All data were analyzed using the SPSS software for Windows (SPSS, Chicago, IL, USA), and $p<0.05$ was considered statistically significant. Continuous data are expressed as a mean \pm standard deviation or median with range and compared by Student's $t$ test or the Mann-Whitney $U$ test. The categorical variables were subject to univariate analysis using the chi-squared test or Fisher's exact test for categorical variables. The multivariate analysis was based on logistic regression. The survival was estimated using the Kaplan-Meier method and defined as the time elapsed from MCS date to death or last follow-up.

\section{Results}

\section{Patents Characteristics, Diagnoses, and Types of Mechanical Support}

The preoperative patient characteristics are shown in Table 1. The median age of patients with MCS was 8.9 years (range: $0.3-15.9$ years) in the Bi-VAD group $(n=11)$ and 5.6 years (range: $0-19.8$ years) in the ECMO group $(n=25)$. Among eleven patients in the Bi-VAD group, five were supported by a Bi-VAD initially and then maintained until weaning or transplantation. Another five were initially supported by ECMO that was eventually converted to Bi-VAD
Table 1 Preoperative MCS patient characteristics

\begin{tabular}{llll}
\hline Variables & Bi-VAD & ECMO & $p$-value \\
\hline Age (year, median, IQR) & $8.6(7.1-12.7)$ & $2.9(0.4-10.4)$ & 0.126 \\
Gender $(n, \%)$ & & & 0.716 \\
Male & $6(54.5 \%)$ & $9(53.0 \%)$ & \\
Female & $5(45.5 \%)$ & $16(36.0 \%)$ & \\
Weight $(\mathrm{kg}$, median, IQR) & $26.9(25.0-52.7)$ & $12.0(7.1-37.0)$ & 0.080 \\
BSA (m ${ }^{2}$, median, IQR) & $0.98(0.95-1.57)$ & $0.53(0.36-1.22)$ & 0.081 \\
Diagnosis $(n, \%)$ & & & \\
Dilated cardiomyopathy & $4(36.4 \%)$ & $14(56.0 \%)$ & 0.278 \\
Acute fulminant myocarditis & $6(54.5 \%)$ & $5(20.0 \%)$ & 0.056 \\
Stress induced cardiomyopathy & $1(9.1 \%)$ & 0 & \\
Autoimmune cardiomyopathy & 0 & $1(4.0 \%)$ & \\
Congenital tricuspid regurgitation & 0 & $1(4.0 \%)$ & \\
Congenital mitral valve regurgitation & 0 & $1(4.0 \%)$ & \\
Persistent pulmonary artery hypertension & 0 & $1(4.0 \%)$ & \\
Pulmonary vein stenosis & 0 & $1(4.0 \%)$ & \\
Unknown origin & 0 & $1(4.0 \%)$ & 0.678 \\
Preoperative renal impairment $(n, \%)$ & $3(27.3 \%)$ & $5(20.0 \%)$ & 0.718 \\
Preoperative hepatic impairment $(n, \%)$ & $7(63.6 \%)$ & $13(52.0 \%)$ & \\
Left ventricle decompression $(n, \%)$ & & $13(52.0 \%)$ & \\
\hline
\end{tabular}

$B i$-VAD bi-ventricular assist device, $B S A$ body surface area, ECMO extracorporeal membranous oxygenator, $I Q R$ interquartile range, $M C S$ mechanical circulartory support, $n$ number of cases 
after median 20.8 days (IQR: 6.0-36.3 days) of ECMO support, and the other one was supported by ECMO initially, and then converted with LVAD after pulmonary function was restored, however, eventually RVAD was added. In the ECMO group, there was no case that was supported by BiVAD initially and then was converted to ECMO support in the end. We added a RVAD 7 days and 33 days after prior LVAD support, respectively, in 2 cases.

The median body weight was $25.5 \mathrm{~kg}$ (range: $2.9-73.3 \mathrm{~kg}$ ). Diagnoses included dilated cardiomyopathy (DCMP, $n=18,50 \%)$, myocarditis $(n=11,30.6 \%)$, and others $(n=7,19.4 \%)$; two cases of cardiomyopathy from autoimmune origin and stress-induced by chemotherapy, two cases of acutely progressive cardiac dysfunction by congenital valvular diseases (tricuspid regurgitation and mitral regurgitation), 1 case of acute biventricular dysfunction accompanying with persistent pulmonary artery hypertension, 1 case of acutely aggravated right heart failure with pulmonary edema accompanying with viral pneumonia and significant pulmonary vein stenosis that had been progressed after repair of total anomalous pulmonary venous return for several months, and 1 case of acute right heart dysfunction accompanying with tricuspid regurgitation and borderline left ventricular dysfunction that we failed to find out specific reasons for this patient's cardiac dysfunction.

Renal impairment was found preoperatively in 3 subjects from the Bi-VAD group and 5 from the ECMO group $(p=0.678)$. Preoperative hepatic impairment was present in 7 patients from the Bi-VAD group and 13 from the ECMO group $(p=0.718)$. Clinical features before MCS are shown in Table 2. Three patients in the Bi-VAD group and 10 patients in the ECMO group were supported by a mechanical ventilator before the operation $(p=0.708)$. There were no differences in metabolic acidosis, $\mathrm{PaO}_{2}, \mathrm{PaCO}_{2}$, bicarbonate, and base excess between the two groups.

\section{Clinical Outcomes}

Table 3 shows the clinical outcomes of the two groups. We were able to support patients longer with Bi-VAD (median 16 days, IQR: 6-31 days) than with ECMO (median 8 days IQR: 4-26 days), even though this difference did not reach statistical significance $(p=0.813)$. In-hospital mortality was seen in 3 patients $(27.3 \%)$ from the Bi-VAD group and 12 (48.0\%) from the ECMO group ( $p=0.295)$. Patients with Bi-VAD support had similar rates of MCS rate and successful transplantation after MCS $(72.7 \%)$ with patients with ECMO support (68.0\%). Figure 1 shows comparison of overall numbers of the patients who underwent transplantation (including 2 mortality cases, 1 in Bi-VAD group, 1 in ECMO group), who expired, and survived after MCS, from Bi-VAD group (blue) and ECMO group (orange). The total transfusion amount, which was adjusted by body surface area, also did not differ between the two groups. However, the amounts of platelets transfused was significantly different between the two groups; median: $90.6 \mathrm{ml} / \mathrm{m}^{2} /$ day, IQR: $34.6-155.1 \mathrm{ml} / \mathrm{m}^{2} /$ day in Bi-VAD group; median: $163.6 \mathrm{ml} / \mathrm{m}^{2} /$ day, IQR: $101.6-232.0 \mathrm{ml} / \mathrm{m}^{2} /$ day in ECMO group, $p=0.05$, Table 3 ). The hemolysis, that is considered as a positive finding when indirect bilirubin, plasma hemoglobin, or LDH increased, rate was lower in the Bi-VAD group compared with the ECMO group ( $p=0.042$, Table 3 ). The nadir $\mathrm{PaO}_{2}$ was significantly lower in the ECMO group than the Bi-VAD group during the MCS despite the use of an oxygenator (Table 4). The Kaplan-Meier analysis indicated there was no overall survival difference between the two groups during median 12.6 months of follow-up (IQR: 1.0-52.5 months) (Fig. 2a). Mortality was occurred median 31.5 days (IQR: 7.8-1388.0 days) after MCS was started in Bi-VAD group, and median 26.0 days (IQR: 10.5-89.5 days) after MCS started in ECMO group.
Table 2 Clinical features before MCS

\begin{tabular}{llll}
\hline Variables & Bi-VAD & ECMO & $p$-value \\
\hline $\begin{array}{l}\text { Mechanical ventilator support before } \\
\quad \text { MCS }(n, \%)\end{array}$ & $3(27.3 \%)$ & $10(40 \%)$ & 0.708 \\
Right ventricular dysfunction $(n, \%)$ & $9(90 \%)$ & $8(22 \%)$ & 0.007 \\
Metabolic acidosis $(n, \%)$ & $8(72.7 \%)$ & $18(72.0 \%)$ & 1.000 \\
$\mathrm{pH}<7.2(n, \%)$ & $3(27.3 \%)$ & $13(52 \%)$ & 0.277 \\
$\mathrm{PaCO}_{2}(\mathrm{mmHg}$, median, IQR) & $35.9(27.0-39.0)$ & $39.0(29.3-51.9)$ & 0.378 \\
$\mathrm{PaO}_{2}(\mathrm{mmHg}$, median, IQR) & $95.0(61.7-121.0)$ & $61.0(36.0-90.5)$ & 0.049 \\
$\mathrm{Bicarbonate}^{(m m o l / l, ~ m e d i a n, ~ I Q R)}$ & $18.3(12.8-22.7)$ & $14.2(10.0-20.7)$ & 0.292 \\
Base excess $(\mathrm{mmol} / \mathrm{l}$, median, IQR) & $-6.5(-12.5$ to 3.6) & $-11.7(-19.6$ to 2.2) & 0.292 \\
\hline
\end{tabular}

$B i$-VAD bi-ventricular assist device, $B S A$ body surface area, ECMO extracorporeal membranous oxygenator, $I Q R$ interquartile range, $M C S$ mechanical circulatory support, $n$ number of cases 
Table 3 Clinical outcomes between Bi-VAD group and ECMO group
Fig. 1 Comparison of number of patients of transplantation, mortality and survival after mechanical cardiac support between Bi-VAD group and ECMO group. $B i$-VAD biventricular ventricular assist device, ECMO extracorporeal membranous oxygenator

\begin{tabular}{llll}
\hline Variables & Bi-VAD & ECMO & $p$-value \\
\hline MCS duration (days, median, IQR) & $16(6-31)$ & $8(4-26)$ & 0.813 \\
Early Mortality $(n, \%)$ & $3(27.3 \%)$ & $12(48.0 \%)$ & 0.295 \\
Weaning $(n, \%)$ & $4(36.4 \%)$ & $10(40.0 \%)$ & 1.000 \\
Transplantation $(n, \%)$ & $4(36.4 \%)$ & $7(28.0 \%)$ & 0.703 \\
Weaning or transplantation & $8(72.7 \%)$ & $17(68.0 \%)$ & 1.000 \\
CRRT $(n, \%)$ & $2(20.0 \%)$ & $15(60.0 \%)$ & 0.060 \\
Transfusion amount $\left(\mathrm{ml} / \mathrm{m}^{2} /\right.$ day, median, IQR) & $398.8(155.1-603.4)$ & $453.3(240.4-856.1)$ & 0.513 \\
RBC & $216.2(97.8-371.5)$ & $175.0(131.4-420.9)$ & 0.913 \\
FFP & $56.6(12.9-149.1)$ & $46.3(19.5-161.8)$ & 0.942 \\
Platelet & $90.6(34.6-155.1)$ & $163.6(101.6-232.0)$ & 0.050 \\
Cryoprecipitate & $0.0(0.0-0.2)$ & $1.3(0.0-38.0)$ & 0.114 \\
Complications $(n, \%)$ & $8(72.7 \%)$ & $10(40.0 \%)$ & 0.070 \\
Bleeding & $6(54.5 \%)$ & $7(28.0 \%)$ & 0.153 \\
Thrombus & $4(36.4 \%)$ & $5(20.0 \%)$ & 0.409 \\
Stroke & $2(18.2 \%)$ & $1(4.0 \%)$ & 0.216 \\
Embolism & $3(27.3 \%)$ & $1(4.0 \%)$ & 0.076 \\
Vascular complication & $0(0 \%)$ & $1(4.0 \%)$ & 1.000 \\
Infection & $0(0 \%)$ & $5(20.0 \%)$ & 0.295 \\
Hemolysis $(n, \%)$ & $3(27.3 \%)$ & $16(64.0 \%)$ & 0.042 \\
DIC $(n, \%)$ & $6(54.5 \%)$ & $18(72.0 \%)$ & 0.446 \\
\hline
\end{tabular}

$B i$-VAD bi-ventricular assist device, $B S A$ body surface area, $C R R T$ continuous renal replacement therapy, $D I C$ disseminated intravascular coagulation, $E C M O$ extracorporeal membranous oxygenator, $F F P$ fresh frozen plasma, $I Q R$ interquartile range, $M C S$ mechanical circulatory support, $n$ number of cases, $R B C$ red blood cells

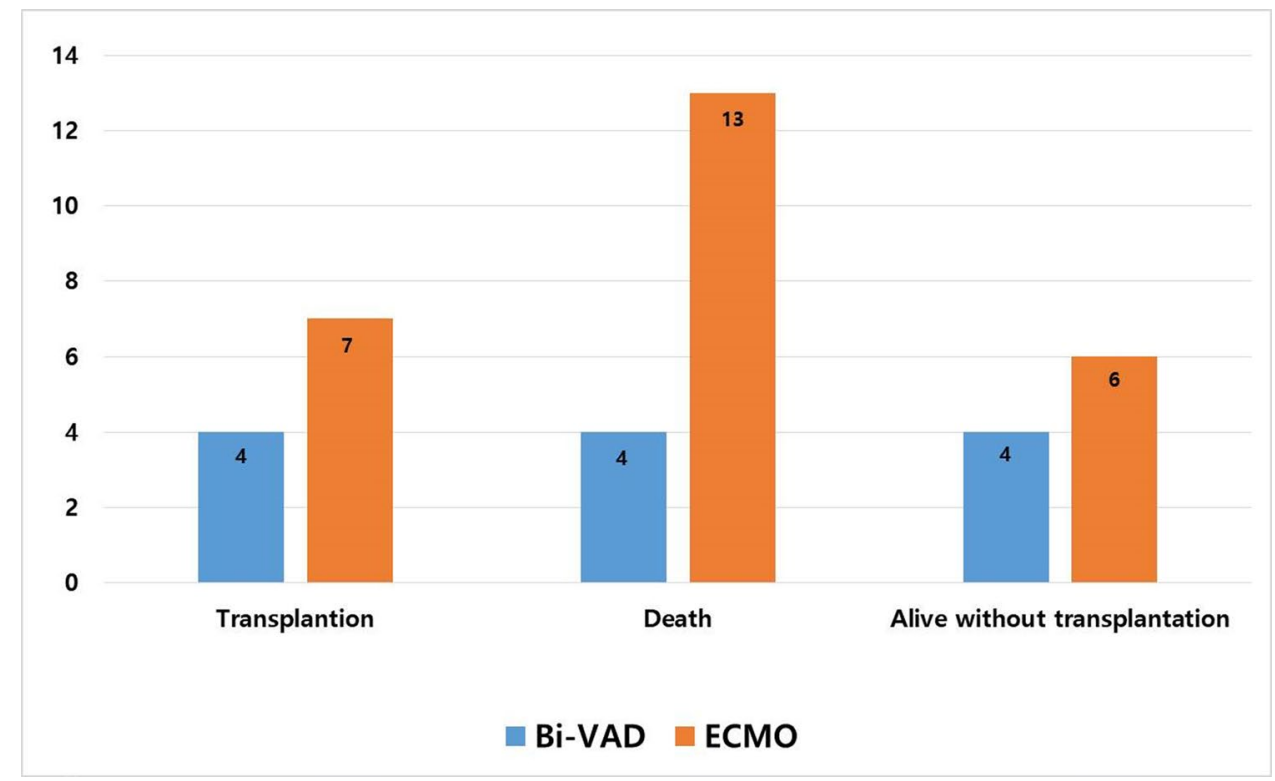

\section{Analysis of Factors for Successful Weaning from MCS and Successful Support}

Several parameters were compared and analyzed between the two groups, patients who had successfully weaned from MCS and patients who failed to wean from MCS or heart transplantation (Table 5). The patients with DCMP $(p=0.006)$ and heart failure before MCS $(p=0.011)$ had a lower rate of successful weaning. Peak creatinine $(p=0.008)$ and BUN $(p<0.001)$ levels were also significantly different between the successfully weaned and failure to wean groups. The multivariable analysis 
Table 4 Laboratory findings during the MCS

\begin{tabular}{lllc}
\hline Variables (median, IQR) & Bi-VAD & ECMO & $p$-value \\
\hline Peak lactate $(\mathrm{mg} / \mathrm{dl})$ & $9.3(4.5-14.5)$ & $10.2(4.7-13.3)$ & 0.892 \\
Nadir pH & $7.20(7.13-7.29)$ & $7.18(7.00-7.28)$ & 0.520 \\
Nadir $\mathrm{PaO}_{2}(\mathrm{mmHg})$ & $80.0(56.8-90.6)$ & $53.2(43.0-71.0)$ & 0.003 \\
Peak BUN (mg/dl) & $51.0(35.0-61.0)$ & $36.0(20.5-64.0)$ & 0.378 \\
Peak creatinine (mg/dl) & $0.90(0.57-2.66)$ & $0.98(0.65-1.66)$ & 0.919 \\
Peak AST (IU/l) & $446.0(135.0-3970.0)$ & $598.0(256.0-2423.5)$ & 0.839 \\
Peak ALT (IU/l) & $155.0(67.0-1677.0)$ & $476.0(120.0-1348.0)$ & 0.588 \\
Peak bilirubin (mg/dl) & $5.1(1.4-18.7)$ & $8.5(3.2-23.9)$ & 0.276
\end{tabular}

$A S T$ aspartate aminotransferase, $A L T$ alanine aminotransferase, $B i$-VAD bi-ventricular assist device, $B U N$ blood urea nitrogen, $E C M O$ extracorporeal membranous oxygenator, $I Q R$ interquartile range, $M C S$ mechanical circulatory support, $R B C$ red blood cells
A

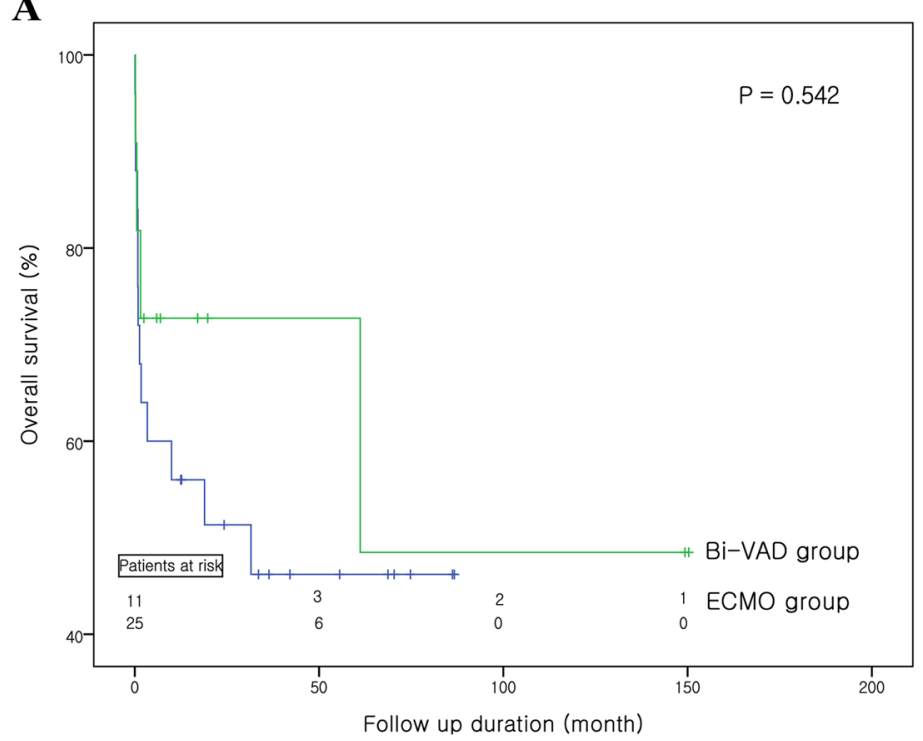

B

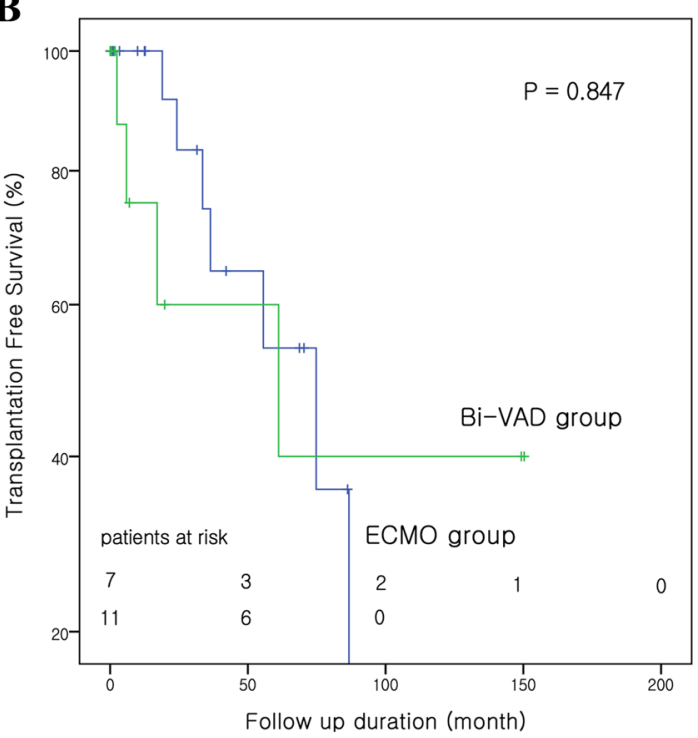

Fig. 2 Kaplan-Meier analysis of a overall survival after MCS weaning or transplantation; $\mathbf{b}$ overall freedom from transplantation. $B i-V A D$ biventricular assist device, ECMO extracorporeal membranous oxygenator, $M C S$ mechanical circulatory support

showed that the patients with DCMP had a significantly lower rate of weaning successfully $(p=0.038$, HR 0.021 [0.001-0.815]).

When we considered "heart transplantation after MCS support" and "successful weaning" as an "eventual successful MCS", although the numbers did not reach statistical significance, we had a greater proportion of patients with myocarditis successfully weaned from MCS or successfully underwent transplantation after MCS (10 of 11, 90.9\%). Regarding dilated cardiomyopathy, though we have small numbers of patients who were supported by Bi-VAD initially, we have more patients who were supported by $\mathrm{Bi}$ VAD from the beginning of the mechanical support in the successful intervention group (11.1\%) than the failed intervention group $(2.8 \%)$.

\section{Outcomes by Era}

There were 10 cases of MCS before 2010 and 26 after 2010 . Figure 3 shows the case numbers of MCS after 2000. After 2010, complication rate of MCS was significantly decreased $(p=0.026)$. Bleeding complications were particularly lower than before $2010(p=0.018)$. The early morality rate tended to improve after 2010, but it did not reach statistical significance $(p=0.058)$. Analyses of outcomes by era are shown in Table 6 . 
Table 5 Analyses of weaning factors after MCS

\begin{tabular}{|c|c|c|c|}
\hline Variables & Weaning & Weaning failure & $p$-value \\
\hline Myocarditis $(n, \%)$ & $7(50.0 \%)$ & $4(18.2 \%)$ & 0.067 \\
\hline Dilated cardiomyopathy $(n, \%)$ & $3(21.4 \%)$ & $15(68.2 \%)$ & 0.006 \\
\hline Heart failure before MCS $(n, \%)$ & $1(7.1 \%)$ & $11(50.0 \%)$ & 0.011 \\
\hline Mechanical ventilator before $\operatorname{MCS}(n, \%)$ & $6(42.9 \%)$ & $7(31.8 \%)$ & 0.501 \\
\hline Previous cardiac surgery $(n, \%)$ & $1(7.1 \%)$ & $4(18.2 \%)$ & 0.628 \\
\hline Bi-VAD first $(n, \%)$ & $1(7.1 \%)$ & $4(18.2 \%)$ & 0.628 \\
\hline Metabolic acidosis before MCS $(n, \%)$ & $10(71.4 \%)$ & $16(72.7 \%)$ & 1.000 \\
\hline Initial creatinine $>1.5 \mathrm{mg} / \mathrm{dl}(n, \%)$ & $3(23.1 \%)$ & $5(22.7 \%)$ & 1.000 \\
\hline Nadir pH (median, IQR) & $7.22(7.10-7.38)$ & $7.27(6.97-7.27)$ & 0.102 \\
\hline Peak Creatinine (mg/dl, median, IQR) & $0.66(0.52-1.00)$ & $1.26(0.79-2.61)$ & 0.008 \\
\hline Peak BUN (ml/dl, median, IQR) & $21.0(16.7-35.2)$ & $55.0(38.7-67.2)$ & 0.000 \\
\hline Peak AST (IU/l, median, IQR) & $482.0(294.3-3883.0)$ & $785.5(294.3-3883.0)$ & 0.150 \\
\hline Peak ALT (IU/l, median, IQR) & $129.0(66.8-551.5)$ & $720.5(163.5-1830.3)$ & 0.038 \\
\hline Peak total bilirubin (mg/dl, median, IQR) & $3.4(1.4-12.2)$ & $8.7(4.2-35.5)$ & 0.045 \\
\hline Total transfusion $\left(\mathrm{ml} / \mathrm{m}^{2} /\right.$ day, median, IQR) & $596.0(215.5-1159.2)$ & $386.4(218.4-651.6)$ & 0.309 \\
\hline $\mathrm{RBC}$ & $311.2(150.8-742.1)$ & $171.1(85.9-281.6)$ & 0.063 \\
\hline FFP & $76.2(6.1-152.9)$ & $47.5(19.2-166.8)$ & 0.817 \\
\hline Platelet & $177.6(54.1-230.7)$ & $138.9(78.8-201.7)$ & 0.736 \\
\hline \multirow[t]{2}{*}{ Cryoprecipitate } & $0.9(0.0-42.9)$ & $0(0-11.8)$ & 0.488 \\
\hline & \multicolumn{3}{|l|}{ Multivariable } \\
\hline Variable & $\mathrm{HR}[95 \% \mathrm{CI}]$ & $p$-value & \\
\hline DCMP & $0.021[0.001-0.815]$ & 0.038 & \\
\hline
\end{tabular}

$A S T$ aspartate aminotransferase, $A L T$ alanine aminotransferase, $B i$-VAD bi-ventricular assist device, $B U N$ blood urea nitrogen, $D C M P$ dilated cardiomyopathy, ECMO extracorporeal membranous oxygenator, $H R$ hazard ratio, $I Q R$ interquartile range, $M C S$ mechanical circulatory support, $n$ number of cases, $R B C$ red blood cells

Fig. 3 Case numbers of mechanical circulatory support since 2000. Bi-VAD biventricular assist device, ECMO extracorporeal membranous oxygenator, $M C S$ mechanical circulatory support

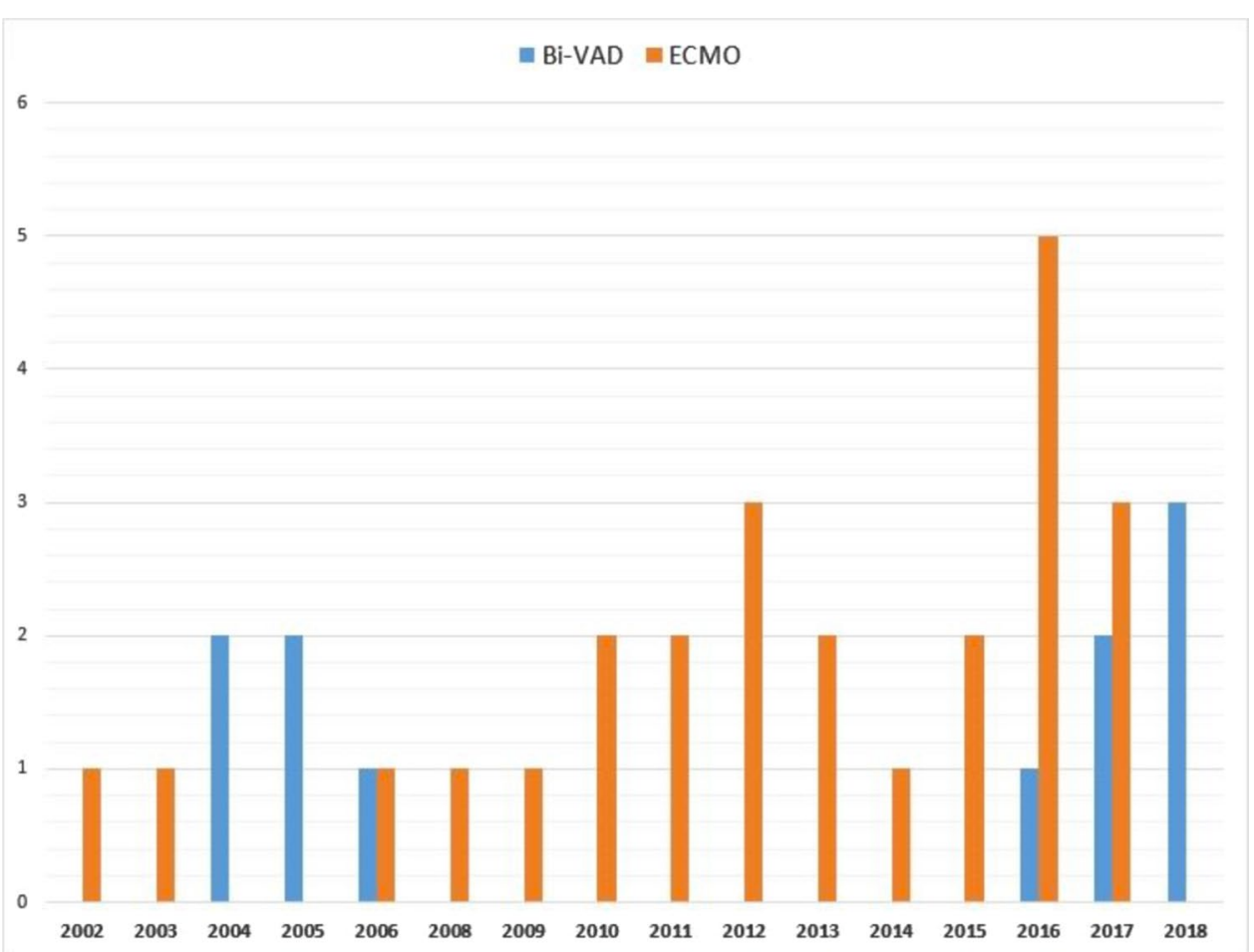


Table 6 Analyses of outcomes by era

\begin{tabular}{llll}
\hline Variables & Before 2010 & After 2010 & P-value \\
\hline Early mortality & $7(70.0 \%)$ & $8(30.8 \%)$ & 0.058 \\
Late mortality & $1(10.0 \%)$ & $1(3.8 \%)$ & 0.484 \\
MCS duration (day, median, IQR) & $12.0(5.8-22.0)$ & $9.5(4.8-31.5)$ & 0.413 \\
Complication (n, \%) & $8(80.0 \%)$ & $10(38.5 \%)$ & 0.026 \\
Bleeding & $7(70.0 \%)$ & $6(23.1 \%)$ & 0.018 \\
Thrombus & $2(20.0 \%)$ & $7(26.0 \%)$ & 1.000 \\
Stroke & $1(10.0 \%)$ & $2(7.7 \%)$ & 1.000 \\
Embolism & $1(10.0 \%)$ & $3(11.5 \%)$ & 1.000 \\
Vascular complication & $1(10.0 \%)$ & $0(0 \%)$ & 0.278 \\
Infection & $3(30.0 \%)$ & $2(7.7 \%)$ & 0.119 \\
Hemolysis $(n, \%)$ & $4(40.0 \%)$ & $15(57.7 \%)$ & 0.463 \\
Disseminated intravascular coagulation (DIC) & $6(60.0 \%)$ & $18(69.2 \%)$ & 0.700 \\
Total transfusion (ml/m ${ }^{2} /$ day, median, IQR) & $583.0(387.7-1386.8)$ & $386.4(208.9-836.4)$ & 0.120 \\
RBC & $323.4(193.7-867.4)$ & $166.4(89.2-312.7)$ & 0.039 \\
FFP & $137.8(65.4-506.6)$ & $35.0(12.3-113.3)$ & 0.026 \\
Platelet & $138.9(78.4-241.2)$ & $145.6(57.9-226.8)$ & 0.935 \\
Cryoprecipitate & $0(0-23.8)$ & $0(0-25.5)$ & 0.827 \\
\hline
\end{tabular}

$D I C$ disseminated intravascular coagulation, FFP fresh frozen plasma, $I Q R$ interquartile range, $M C S$ mechanical circulatory support, $R B C$ red blood cells

\section{Discussion}

This study investigated differences between the clinical outcomes of temporary centrifugal pump-based Bi-VAD and ECMO as rescue treatments for medically intractable acute decompensated heart failure in pediatric patients. Traditionally, to support small pediatric patients with medically intractable heart failure, ECMO is preferred over a VADbased system even when the patients have no respiratory issues and do not require an oxygenator; the limitations that underlie this treatment choice are largely associated with the challenges of applying VAD systems to small patients. For example, VADs require a central approach via sternotomy and this can induce more bleeding complications than the peripheral approach frequently used for ECMO insertion. For the Bi-VAD specifically, the operative field is particularly complicated, especially in small patients, as at least four cannulae are required.

However, in 2016, we started favoring a centrifugal pump-based VAD over ECMO for its longevity and to avoid the complications associated with ECMO, in cases that do not require immediate peripheral vessel approaches. Even though the ECMO was applied initially, after the patient's respiratory function recovered and no longer required oxygenator support, we tried to change the ECMO to a VAD as soon as possible. This approach was also applied in cases of pediatric patients' $\mathrm{RV}$ dysfunction appearing or progressing after LVAD insertion despite nitric oxide gas or milrinone treatment. If the patient's lung condition allowed, we added a RVAD rather than an ECMO for RV support.
Implantable VADs have only been approved for use in pediatric patients in Korea since 2017, and the medical insurance system still does not provide sufficient financial support for the patient's family. Therefore, implantable VAD is still not a straightforward option in Korea. Given this situation, applying a temporary VAD using centrifugal pumps has some financial advantages than immediately applying the implantable is more durable than ECMO-based system because we generally maintain the MCS in the presence of low levels of anticoagulants in the VAD-based system, which results in less hemorrhagic, fewer hemolytic complications and smaller transfusion volumes.

Previous studies reported that the Bi-VAD represents a significant risk factor for poor outcomes [7, 11, 13] because adding a RVAD after LVAD insertion means that the patients' right heart is unable to endure the preload produced by the mechanically-assisted left heart, even with ample pharmaceutical support for right heart. For the present study, we chose a different approach that involved applying the RVAD earlier than previous studies described. When a patient with no major respiration issues began to present with RV dysfunction despite continued medical treatment, we chose to add the RVAD rather than adding more medicine. Given that the aggravation of RV dysfunction after LVAD support over time is not uncommon [7-9], some authors have emphasized the importance of appropriately timing unloading both ventricles for successfully bridging to recovery $[15,16]$. We expected that our early Bi-VAD application strategy would lead to better outcomes in terms of successfully bridging to recovery 
or transplantation, with lower complication and mortality rates than ECMO-treated patients; however, there were no significant differences in the mortality rate and weaning rates between the Bi-VAD and ECMO groups in this study. This might be due to the small number of patients for whom this strategy was applied since our early RVADapply strategy has only been used for 3 to 4 years. Repeating this analysis in the future on a larger patient cohort should provide more statistically meaningful information regard the effect of our strategy on patient outcomes, because, in terms of hemolysis and cryoprecipitate transfusion amounts, the Bi-VAD group required significantly less than the ECMO group, despite the small sample size. We also found that rates of early mortality, bleeding complications, and RBC, FFP transfusion amounts tended to improve by time (Table 6 ). While there are certainly still numerous clinical challenges facing the patients supported by MCS, the accumulation of MCS management experience, development of equipment and systems in intensive care units, and technological advances are making those obstacles more surmountable one small step at a time. Among many factors, the timing of introducing adequate MCS represents one of the most important elements for improving outcomes in these patients.

Levin et al. reported that preoperative hepatic and renal dysfunction were important factors in the requirement of Bi-VAD at the beginning of mechanical support [17]. In our study, patients with hepatic and renal dysfunction that progressed during the MCS had lower rates of weaning compared with patients without these complications; however, preoperative hepatic and renal dysfunction were not risk factors for Bi-VAD-based intervention. This could be due, in part at least, to our approach of implanting the RVAD earlier than previous studies, namely, before hepatic or renal function became aggravated; however, it could also be that earlier application of the RVAD, or Bi-VAD support, might prevent the development of renal or hepatic dysfunction, and this could lead to improved weaning rates or successfully bridging to next treatment stage, (i.e., heart transplantation).

Implantable VAD systems are an effective treatment for heart failure. This study showed that a temporary centrifugal pump-based Bi-VAD remains relevant in selected cases of medically intractable acute decompensated heart failure, particularly in patients with myocarditis with acceptable pulmonary function. It enables effective biventricular unloading for myocardial recovery while buying additional time to determine the best-suited treatment option (i.e., an implantable VAD system or transplantation) and is relatively cost-effective. Especially when treating myocarditis, it represents an affordable option for recovery before considering the implantable VAD system as the recovery period of this particular disease is shorter than most other causes of heart failure.
This retrospective, non-randomized, single-center study has several limitations. The small size of the sample population prevented high power statistical analyses and restricted the interpretation of the results. Regardless, we were nonetheless able to establish that the rapid application of a temporary centrifugal pump-based Bi-VAD was not clinically inferior to the traditional ECMO-based treatment regimen in terms of pediatric patients. Furthermore, our findings illuminated some benefits and advantages including the decreased hemolysis and transfusion required with the $\mathrm{Bi}$ VAD approach. Based on these results, we will continue to employ our strategy of early RVAD addition for patients already supported by LVAD with acceptable levels of respiratory function when the patient initially shows signs of $\mathrm{RV}$ deterioration instead of increasing medical treatment efforts up to its' maximal level.

\section{Compliance with Ethical Standards}

Conflicts of interest None.

\section{References}

1. Osaki S, Edwards NM, Velez M, Johnson MR, Murray MA, Hoffmann JA, Kohmoto T (2008) Improved survival in patients with ventricular assist device therapy: the University of Wisconsin experience. Eur J Cardiothorac Surg 34:281-288

2. Rossano JW, Jang GY (2015) Pediatric heart failure: current state and future possibilities. Korean Circ J 45:1-8

3. Bakhtiary F, Keller H, Dogan S, Dzemali O, Oezaslan F, Meininger D, Ackermann H, Zwissler B, Kleine P, Moritz A (2008) Venoarterial extracorporeal membrane oxygenation for treatment of cardiogenic shock: clinical experiences in 45 adult patients. J Thorac Cardiovasc Surg 135:382-388

4. Fuchs A, Netz H (2001) Ventricular assist devices in pediatrics. Images Paediatr Cardiol 3:24-54

5. Miller JR, Epstein DJ, Henn MC, Guthrie T, Schuessler RB, Simpson KE, Canter CE, Eghtesady P, Boston US (2015) Early biventricular assist device use in children: a single-center review of 31 patients. ASAIO J 61:688-694

6. Fraser CD, Jr., Jaquiss RD, Rosenthal DN, Humpl T, Canter CE, Blackstone EH, Naftel DC, Ichord RN, Bomgaars L, Tweddell JS, Massicotte MP, Turrentine MW, Cohen GA, Devaney EJ, Pearce FB, Carberry KE, Kroslowitz R, Almond CS, Berlin Heart Study I (2012) Prospective trial of a pediatric ventricular assist device. N Engl J Med 367:532-541

7. Almond CS, Morales DL, Blackstone EH, Turrentine MW, Imamura M, Massicotte MP, Jordan LC, Devaney EJ, Ravishankar C, Kanter KR, Holman W, Kroslowitz R, Tjossem C, Thuita L, Cohen GA, Buchholz H, St Louis JD, Nguyen K, Niebler RA, Walters HL 3rd, Reemtsen B, Wearden PD, Reinhartz O, Guleserian KJ, Mitchell MB, Bleiweis MS, Canter CE, Humpl T (2013) Berlin Heart EXCOR pediatric ventricular assist device for bridge to heart transplantation in US children. Circulation 127:1702-1711

8. Cassidy J, Dominguez T, Haynes S, Burch M, Kirk R, Hoskote A, Smith J, Fenton M, Griselli M, Hsia TY, Ferguson L, Van Doorn C, Hasan A, Karimova A (2013) A longer waiting game: 
bridging children to heart transplant with the Berlin Heart EXCOR device: the United Kingdom experience. J Heart Lung Transplant 32:1101-1106

9. Morales DL, Almond CS, Jaquiss RD, Rosenthal DN, Naftel DC, Massicotte MP, Humpl T, Turrentine MW, Tweddell JS, Cohen GA, Kroslowitz R, Devaney EJ, Canter CE, Fynn-Thompson F, Reinhartz O, Imamura M, Ghanayem NS, Buchholz H, Furness S, Mazor R, Gandhi SK, Fraser CD Jr (2011) Bridging children of all sizes to cardiac transplantation: the initial multicenter North American experience with the Berlin Heart EXCOR ventricular assist device. J Heart Lung Transplant 30:1-8

10. Blume ED, Naftel DC, Bastardi HJ, Duncan BW, Kirklin JK, Webber SA, Pediatric Heart Transplant Study I (2006) Outcomes of children bridged to heart transplantation with ventricular assist devices: a multi-institutional study. Circulation 113:2313-2319

11. Stein ML, Robbins R, Sabati AA, Reinhartz O, Chin C, Liu E, Bernstein D, Roth S, Wright G, Reitz B, Rosenthal D (2010) Interagency Registry for Mechanically Assisted Circulatory Support (INTERMACS)-defined morbidity and mortality associated with pediatric ventricular assist device support at a single US center: the Stanford experience. Circ Heart Fail 3:682-688

12. Jeewa A, Manlhiot C, McCrindle BW, Van Arsdell G, Humpl T, Dipchand AI (2010) Outcomes with ventricular assist device versus extracorporeal membrane oxygenation as a bridge to pediatric heart transplantation. Artif Organs 34:1087-1091

13. Karimova A, Pockett CR, Lasuen N, Dedieu N, Rutledge J, Fenton M, Vanderpluym C, Rebeyka IM, Dominguez TE, Buchholz H
(2014) Right ventricular dysfunction in children supported with pulsatile ventricular assist devices. J Thorac Cardiovasc Surg 147:1691-1697

14. Rockett SR, Bryant JC, Morrow WR, Frazier EA, Fiser WP, McKamie WA, Johnson CE, Chipman CW, Imamura M, Jaquiss RD (2008) Preliminary single center North American experience with the Berlin Heart pediatric EXCOR device. ASAIO J 54:479-482

15. Saito S, Toda K, Miyagawa S, Yoshikawa Y, Hata H, Yoshioka D, Domae K, Tsukamoto Y, Sakata Y, Sawa Y (2018) Diagnosis, medical treatment, and stepwise mechanical circulatory support for fulminat myocarditis. J Artif Organs 21:172-179

16. Ranganath NK, Smith DE, Moazami N (2018) The Achilles' heel of left ventricular assist device therapy: right ventricle. Curr Opin Organ Transplant 23:295-300

17. Levin AP, Jaramillo N, Garan AR, Takeda K, Takayama H, Yuzefpolskaya M, Mancini DM, Naka Y, Colombo PC, Topkara VK (2016) Outcomes of contemporary mechanical circulatory support device configurations in patients with severe biventricular failure. J Thorac Cardiovasc Surg 151:530-535

Publisher's Note Springer Nature remains neutral with regard to jurisdictional claims in published maps and institutional affiliations. 